\title{
FIRST RECORDS OF BATS (Mammalia: Chiroptera) FROM SON LA CITY, NORTHWESTERN VIETNAM, WITH REMARKS ON THEIR CONSERVATION STATUS
}

\author{
Dao Nhan Loi \\ Faculty of Agriculture and Forestry, Tay Bac University, Son La, Vietnam \\ Received 19 May 2019, accepted 20 August 2019
}

\begin{abstract}
Son $\mathrm{La}$ is a well-known city in the Northwestern region of Vietnam for its outstanding landscapes. It contains a variety of ecosystems ranging from farming area, valleys, forests on hills and karst with cave systems. Natural habitats of the city would be suitable for many bat species. However, bats of the city were not included in any literature. Between March 2014 and June 2018, we conducted a series of bat surveys in this city using mist nets and harp traps. Results from our study indicated that Son La city is a home to at least 21 bat species belonging to 9 genera, 6 families. Of which, Rhinolophus thomasi was listed as "VU" (Vulnerable) in the 2007 Red Book of Vietnam and Myotis annamiticus was listed in the IUCN Redlist of the Threatened Species as "DD" (Data Deficient). This paper provides the first data on bats of Son La city with morphological measurements and conservation status of each recorded species.
\end{abstract}

Keywords: Biodiversity, conservation, mammal, northwestern Vietnam.

Citation: Dao Nhan Loi, 2019. First records of bats (Mammalia: Chiroptera) from Son La city, Northwestern Vietnam, with remarks on their conservation status. Academia Journal of Biology, 41(3): 123-127. https://doi.org/10.15625/2615-0923/v41n3.13791.

*Corresponding author email: daonhanloi@ gmail.com

(C2019 Vietnam Academy of Science and Technology (VAST) 


\section{INTRODUCTION}

Son La city, the capital of Son La Province, is one of the most important sites within the northwestern region of Vietnam for both nature conservation and socio-economic development. The first assessment of mammal diversity from the province was published by Tran Hong Viet et al. (2007a) which included records of 35 bat species belonging to 18 genera, 8 families. However, taxonomic status of several species were recently changed while some species were unconfirmed. Dao Nhan Loi (2017) provided an assessment of bat diversity of Son La province with records of 43 bat species belonging to 19 genera, 5 families. This updated assessment comprises original data from field surveys and information from previously published records of bats from Thuan Chau district (13 species, 9 genera, 5 families), Quynh Nhai (13 species, 9 genera, 4 families), Moc Chau (10 species, 7 genera, 4 families), Yen Chau (13 species, 8 genera, 6 families), Muong Do and Phu Yen (37 species, 18 genera, 8 families) (Tran Hong Viet et al. 2006a, 2006b, 2006c, 2006d; Tran Hong Hai et al. 2010). The above results indicated that Son La province is a hotspot in Vietnam for bat research and conservation. However, the previous mammal surveys were only carried out in protected areas and districts of the province. Bats and other animals of many areas including the Son La city had not received attention from scientist by 2013. Between 2014 and 2018, the author conducted field surveys throughout the habitats of the city and obtained the first data of bats of the Son La city. Many species, which were nationally rare but commonly recorded in Son La city. It is likely that the city contains important remaining habitats for bat species. This paper provides current information regarding diversity, distribution and conservation status of each bat species at the city.

\section{MATERIALS AND METHODS}

Field surveys were carried out during 109 days at seven selected communes of the Son La city: Chieng Sinh, Chieng Coi, Quyet Tam, Chieng Ngan wards, Chieng Xom, Chieng
An, Hua La communes (Fig. 1). Bats were captured using mist nets in different sizes (10.0 m x $2.5 \mathrm{~m} ; 12.0 \mathrm{~m} \times 3.0 \mathrm{~m} ; 7.0 \mathrm{~m} \times 2.5$ $\mathrm{m})$ and harp traps. The nets and traps were set up under forest canopy, cave entrances, above the water bodies (streams and lakes) and other habitats. The nets were open from 5:30 pm to around 11:00 pm. The traps were often placed across trails in the forest, cave doors or trails under forest canopy. A total of 142 individuals were captured over the surveys. The following morphological mesurements of every captured individuals were taken in the field for rapid identification: forearm length (FA), ear height (EH), Tibia length (TIB), hind foot length (HF), tail length (T). These measurements were illustrated in Bates \& Harison (1997). Pregnant or lactating females were released immediately after quick taking of the above measurments. Selected individuals were kept as specimens which are preserved at Faculty of Agriculture and Forestry, Tay Bac University, Son La city, and the Institute of Ecology and Biological Resources, VAST, Ha Noi.

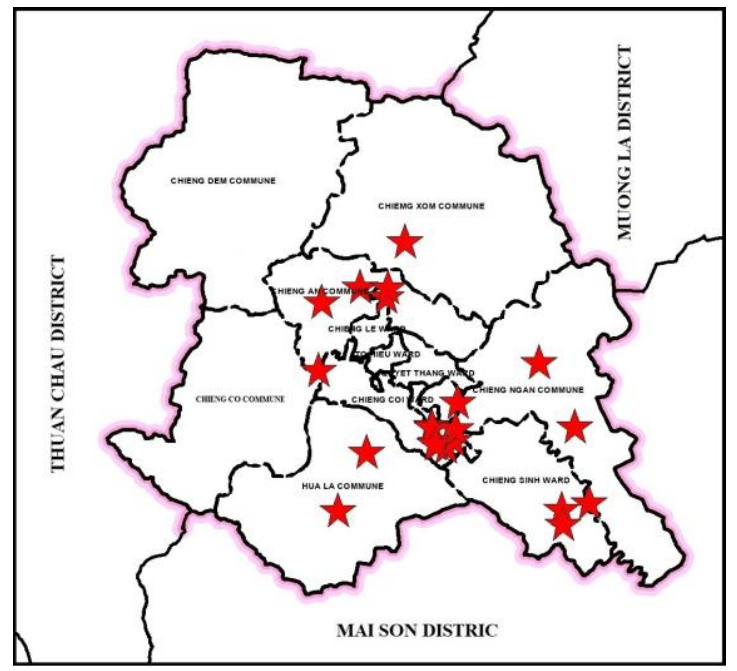

Figure 1. Study sites within the Son La city

Representatives of each species was kept as voucher specimen for craniodental characteristics to confirm their taxonomic status. All voucher specimens were identified with reference to the publications related to 
the bat fauna of Vietnam (Corbet \& Hill 1992; Brissenko \& Kruskov 2003; Csorba et al. 2003; Kruskop 2013).

\section{RESULTS AND DISCUSSION}

Results from the surveys suggested that the Son La city is home to at least 21 bat species belonging to 9 genera, 6 families (table 1). Of which, 2 species are listed in the
2007 Red Data Book of Vietnam: Rhilophus thomasi and Myotis siligorensis. Almost all remaining species are listed as LC (Least concern) in the current IUCN Red List of the Threatened Species except Myotis annamiticus which is listed as DD (data definicient) and an unassessed species (Tylonycteris fulvida).

Table 1. Diversity and conservation status of bat species recorded from Son La city

\begin{tabular}{|c|c|c|c|c|}
\hline \multirow{2}{*}{ Scientific name } & \multirow{2}{*}{ English name } & \multirow{2}{*}{ Vietnamese name } & \multicolumn{2}{|c|}{ Conservation status } \\
\hline & & & IUCN & VNRB \\
\hline Pteropodiae & Old World fuit bats & Họ dơi quả & & \\
\hline Megaerops niphanae & Ratanaworabhan's Fruit Bat & Dơi quả không đuôi lớn & $\mathrm{LC}$ & \\
\hline Emballonuridae & Emballonurids & Họ dơi bao đuôi & & \\
\hline Taphozous melanopogon & Black - bearded tomb bat & Dơi bao đuôi nâu đen & $\mathrm{LC}$ & \\
\hline Hipposideridae & Old - World leaf-nosed bats & Họ dơi nếp mũi & & \\
\hline Asellicus stoliczkanus & Stoliczka's trident bat & Dơi mũi ba lá & & \\
\hline Hipposideros larvatus & Horsfield's leaf-nosed bat & Dơi mũi xám & $\mathrm{LC}$ & \\
\hline Hipposideros pomona & Andersen's leaf-nosed bat & Dơi nếp mũi xinh & $\mathrm{LC}$ & \\
\hline Miniopteridae & Bent-winged bats & Họ dơi cánh dài & & \\
\hline Miniopterus fuliginosus & Eastern bent-winged bat & Dơi gập cánh dài & & \\
\hline Rhinolophidae & Horseshoe bats & Họ dơi lá mũi & & \\
\hline Rhinolophus affinis & Intermediate horseshoe bat & Dơi lá đuôi & $\mathrm{LC}$ & \\
\hline Rhinolophus siamensis & Thai Horseshoe Bat & Dơi lá mũi thái lan & & \\
\hline Rhinolophus malayanus & Malayan horseshoe bat & Dơi lá mã lai & $\mathrm{LC}$ & \\
\hline Rhinolophus marshalli & Marshall's horseshoe bat & Dơi lá rẻ quạt & $\mathrm{LC}$ & \\
\hline $\begin{array}{l}\text { Rhinolophus } \\
\text { microglobosus }\end{array}$ & $\begin{array}{l}\text { Indo-Chinese brown } \\
\text { horseshoe bat }\end{array}$ & Dơi lá mũi bắc & $\mathrm{LC}$ & \\
\hline Rhinolophus pearsonii & Pearson's horseshoe bat & Dơi lá pec-xôn & $\mathrm{LC}$ & \\
\hline Rhinolophus pusillus & Least horseshoe bat & Dơi lá muỗi & $\mathrm{LC}$ & \\
\hline Rhinolophus thomasi & Thomas's horseshoe bat & Dơi lá tô - ma & $\mathrm{LC}$ & VU \\
\hline Vespertilionidae & Vesper bat & Họ dơi muỗi & & \\
\hline Myotis annamiticus & Annamite myotis & Dơi tai việt nam & DD & \\
\hline Myotis hasseltii & Lesser Large-footed Myotis & Dơi tai hat-xen & $\mathrm{LC}$ & \\
\hline Myotis horsfieldii & Horsfield's Myotis & Dơi tai cánh ngắn & $\mathrm{LC}$ & \\
\hline Myotis laniger & Chinese Water Myotis & Dơi tai trung hoa & $\mathrm{LC}$ & \\
\hline Myotis siligorensis & Himalayan whiskered bat & Dơi tai sọ cao & $\mathrm{LC}$ & LR \\
\hline Pipistrellus abramus & Japanese pipistrelle & Dơi muỗi sọ dẹt & $\mathrm{LC}$ & \\
\hline Tylonycteris fulvida & Amber Bamboo Bat & Dơi ống tre & N/A & \\
\hline
\end{tabular}

Notes: VNRB: The 2007 Red Data Book of Vietnam; IUCN: IUCN Red List of the Threatened Species (http://www.iucnredlist.org).

Of the recorded bat families, Rhinolophidae comprises the highest species number which accounts for $38.10 \%$ of the total known species (table 2). Four species were commonly recorded at almost all study sites (Rhinolophus thomasi, $R$. siamensis, $R$. 
affinis) while four remaining ones were locally rare ( $R$. malayanus, $R$. marshalli, $R$. pearsonii, $R$. pusillus). Vespertilionidae consists of 7 species, accounting for $33.3 \%$ of all known species from the study area (table 2). Of which, Myotis hordfieldii, Pipistrellus abramus were quite common throughout the city while three species (Tylonycteris fulvida, Myotis siligorensis, $M$. hasseltii) were rarely recorded.

Among the remaining families, Hipposideridae consists of quite common species but three other families (Pteropodidae, Emballonuridae, and Miniopteridae) were uncommon and less diverse (table 2).
Morphological measurements of every captured species are given in the table 3 .

Table 2. Bat species composition from the study area

\begin{tabular}{|l|c|c|c|c|}
\hline \multirow{2}{*}{ Family name } & \multicolumn{2}{c|}{ Genera } & \multicolumn{2}{c|}{ Species } \\
\cline { 2 - 5 } & $\mathrm{n}$ & $\%$ & $\mathrm{n}$ & $\%$ \\
\hline Pteropodiae & 1 & 11.11 & 1 & 4.76 \\
\hline Emballonuridae & 1 & 11.11 & 1 & 4.76 \\
\hline Hipposideridae & 2 & 22.22 & 3 & 14.29 \\
\hline Miniopteridae & 1 & 11.11 & 1 & 4.76 \\
\hline Rhinolophidae & 1 & 11.11 & 8 & 38.10 \\
\hline Vespertilionidae & 3 & 33.33 & 7 & 33.33 \\
\hline Total & 9 & 100.00 & 21 & 100.00 \\
\hline
\end{tabular}

Table 3. External measurements of bat species from the study area

\begin{tabular}{|c|c|c|c|c|c|c|}
\hline \multirow{2}{*}{ Species } & \multirow{2}{*}{$\mathrm{n}$} & \multicolumn{5}{|c|}{ External measurements } \\
\hline & & FA & $\mathrm{EH}$ & TIB & $\mathrm{HF}$ & $\mathrm{T}$ \\
\hline Megaerops niphanae & 3 & $\begin{array}{c}59,27 \pm 2,53 \\
56,43-61,28\end{array}$ & $\begin{array}{c}17,07 \pm 0,76 \\
16,47-17,92\end{array}$ & $\begin{array}{c}23,69 \pm 1,25 \\
22,27-24,63\end{array}$ & $\begin{array}{l}10,00 \pm 0,37 \\
9,73-10,42\end{array}$ & \\
\hline Taphozous melanopogon & 21 & $\begin{array}{c}66,33 \pm 1,38 \\
64,15-68,40\end{array}$ & $\begin{array}{c}14,23 \pm 0,91 \\
13,00-17,51\end{array}$ & $\begin{array}{c}25,31 \pm 0,66 \\
24,20-26,71\end{array}$ & $\begin{array}{c}12,50 \pm 0,30 \\
12,02-13,07\end{array}$ & $\begin{array}{c}27,14 \pm 1,81 \\
22,24-30,10\end{array}$ \\
\hline Aselliscus stoliczkanus & 12 & $\begin{array}{c}43,21 \pm 0,93 \\
41,52-44,73\end{array}$ & $\begin{array}{c}11,88 \pm 0,71 \\
11,14-13,66\end{array}$ & $\begin{array}{c}19,83 \pm 0,85 \\
18,45-21,24\end{array}$ & $\begin{array}{l}7,27 \pm 0,39 \\
6,69-7,97\end{array}$ & $\begin{array}{c}36,20-3,10 \\
29,94-40,38\end{array}$ \\
\hline Hipposideros lavatus & 15 & $\begin{array}{c}59,22 \pm 1,77 \\
54,92-61,78\end{array}$ & $\begin{array}{c}22,25 \pm 2,42 \\
18,38-27,55\end{array}$ & $\begin{array}{c}24,64 \pm 1,20 \\
22,58-26,78\end{array}$ & $\begin{array}{l}10,28 \pm 0,86 \\
9,35-11,63\end{array}$ & $\begin{array}{c}35,63 \pm 2,33 \\
31,67-39,66\end{array}$ \\
\hline H. pomona & 18 & $\begin{array}{c}42,51 \pm 1,08 \\
40,82-45,51\end{array}$ & $\begin{array}{c}21,94 \pm 2,47 \\
15,10-25,83\end{array}$ & $\begin{array}{c}19,70 \pm 1,66 \\
18,03-24,73\end{array}$ & $\begin{array}{l}7,66 \pm 0,59 \\
6,67-8,89\end{array}$ & $\begin{array}{c}31,36 \pm 4,93 \\
17,67-35,89\end{array}$ \\
\hline Miniopterus fuliginosus & 7 & $\begin{array}{c}49,99 \pm 0,83 \\
49,07-51,37\end{array}$ & $\begin{array}{c}12,13 \pm 1,24 \\
10,18-13,35\end{array}$ & $\begin{array}{c}20,89 \pm 0,51 \\
20,21-21,54\end{array}$ & $\begin{array}{l}10,40 \pm 0,54 \\
9,22-10,69\end{array}$ & $\begin{array}{c}57,68 \pm 3,24 \\
53,18-61,13\end{array}$ \\
\hline Rhinolophus affinis & 5 & $\begin{array}{c}53,39 \pm 0,56 \\
52,91-53,84\end{array}$ & $\begin{array}{c}19,69-2,84 \\
16,65-23,43\end{array}$ & $\begin{array}{c}25,40 \pm 0,69 \\
24,59-26,17\end{array}$ & $\begin{array}{c}11,31 \pm 0,35 \\
11,03-11,89\end{array}$ & $\begin{array}{c}26,15 \pm 2,15 \\
22,56-28,30\end{array}$ \\
\hline R. siamensis & 11 & $\begin{array}{c}39,40 \pm 0,99 \\
37,52-40,43\end{array}$ & $\begin{array}{c}22,35 \pm 2,54 \\
20,18-29,69\end{array}$ & $\begin{array}{c}15,63 \pm 1,61 \\
11,00-17,01 \\
\end{array}$ & $\begin{array}{l}7,25 \pm 0,39 \\
6,80-7,93 \\
\end{array}$ & $\begin{array}{c}17,53 \pm 3,41 \\
11,02-21,53\end{array}$ \\
\hline R. malayanus & 1 & 45,89 & 15,14 & 17,42 & 7,42 & 22,83 \\
\hline R. marshalli & 1 & 46,51 & 25,88 & 20,00 & 8,19 & 22,15 \\
\hline R.microglobosus & 4 & $\begin{array}{c}45,60 \pm 1,33 \\
43,66-46,70 \\
\end{array}$ & $\begin{array}{c}16,90 \pm 2,19 \\
14,16-19,36 \\
\end{array}$ & $\begin{array}{c}2,18 \pm 1,41 \\
20,95-23,72\end{array}$ & $\begin{array}{c}9,91 \pm 1,44 \\
8,24-10,78 \\
\end{array}$ & $\begin{array}{r}18,75 \pm 1,10 \\
17,73-19,91 \\
\end{array}$ \\
\hline R. pearsonii & 1 & 53,95 & 27,66 & 25,84 & 11,30 & 18,95 \\
\hline R. pusillus & 1 & 37,76 & 16,38 & 16,62 & 5,77 & 18,19 \\
\hline R. thomasi & 7 & $\begin{array}{c}44,44 \pm 1,64 \\
41,13-46,08\end{array}$ & $\begin{array}{c}17,37 \pm 2,31 \\
14,49-20,34\end{array}$ & $\begin{array}{c}18,60 \pm 0,56 \\
17,90-19,52\end{array}$ & $\begin{array}{l}7,86 \pm 0,71 \\
7,05-8,05\end{array}$ & $\begin{array}{c}22,31 \pm 2,02 \\
20,04-25,86\end{array}$ \\
\hline Myotis annamiticus & 3 & $\begin{array}{c}35,55 \pm 1,63 \\
33,76-36,95\end{array}$ & $\begin{array}{c}12,76 \pm 1,13 \\
11,70-13,95\end{array}$ & $\begin{array}{c}13,97 \pm 1,16 \\
12,98-15,25\end{array}$ & $\begin{array}{l}7,59 \pm 1,44 \\
6,68-9,25\end{array}$ & $\begin{array}{c}35,52 \pm 1,02 \\
34,67-36,65\end{array}$ \\
\hline M. hasseltii & 1 & 35,55 & 12,7 & 13,82 & 7,48 & 38,72 \\
\hline M. hordfieldii & 16 & $\begin{array}{c}35,02 \pm 1,43 \\
31,62-37,75 \\
\end{array}$ & $\begin{array}{c}13,88 \pm 1,72 \\
10,54-15,83\end{array}$ & $\begin{array}{c}13,98 \pm 1,48 \\
10,91-15,95 \\
\end{array}$ & $\begin{array}{c}8,54 \pm 1,29 \\
6,05-10,42 \\
\end{array}$ & $\begin{array}{c}36,98 \pm 3,13 \\
27,60-39,98 \\
\end{array}$ \\
\hline M. laniger & 4 & $\begin{array}{c}35,04 \pm 0,63 \\
34,36-35,67\end{array}$ & $\begin{array}{c}14,40 \pm 0,87 \\
13,30-15,16\end{array}$ & $\begin{array}{c}15,06 \pm 0,43 \\
14,49-15,51 \\
\end{array}$ & $\begin{array}{l}8,17 \pm 0,48 \\
7,58-8,69\end{array}$ & \\
\hline M. siligorensis & 2 & $33,98-34,43$ & $8,94-11,68$ & $13,50-13,68$ & $6,03-7,23$ & $31,85-41,43$ \\
\hline Pipistrellus abramus & 7 & $\begin{array}{c}31,86 \pm 1,07 \\
30,41-33,07\end{array}$ & $\begin{array}{c}9,69 \pm 0,98 \\
8,50-11,13\end{array}$ & $\begin{array}{c}12,20 \pm 0,90 \\
10,60-12,97 \\
\end{array}$ & $\begin{array}{l}6,36 \pm 0,43 \\
5,74-7,13\end{array}$ & $\begin{array}{c}32,46 \pm 2,80 \\
30,10-37,60\end{array}$ \\
\hline Tylonycteris fulvida & 2 & $25,15-25,68$ & $7,86-7,91$ & $11,28-11,90$ & $5,65-5,77$ & $26,67-26,69$ \\
\hline
\end{tabular}

Notes: Values are given as mean $\pm \mathrm{SD}$, minimum-maximum. Abbreviations are defined in the "Material and Methods"; $\mathrm{n}=$ sample size. 


\section{CONCLUSION}

The Son La city is home to at least 21 bat species belonging to 9 genera, 6 families. Of which, two species (Rhinolophus thomasi and Myotis siligorensis) are listed in the 2007 Red Data Book of Vietnam; 16 species are listed as "Least Concern" in the current IUCN Red List of the Threatened Species (IUCN). Myotis annamiticus, which is listed as DD in IUCN, was also recorded from different study sites of Son La city.

\section{REFERENCES}

Bates. P. J. J., Thong. V. D., Bumrungsri. S., 2005. Voucher specimen preparation: bats, Part of the Darwin Initiative Project: Taxonomic initiative for Southeast Asian bat studies (Vietnam, Thailand, Cambodia, and Lao PDR). 12.

Bates. P. J. J., Harison. D. L., 1997. Bats of the Indian Subcontinent. Harrison Zoological Museum publication, Sevenoaks, Kent, United Kingdom, 258.

Brissenko. A. V., Kruskov. S. V., 2003. Bats of Vietnam and adjacent territoriesan identification manual. Moscow, 211.

Dang Ngoc Can, Hideki Endo, Nguyen Truong Son, Tatsuo Oshida, Le Xuan Canh, Dang Checklist of wild mammal species of Vietnam. Ha Noi: Primate Research

Institute, Inuyama, Japan and Institute of Ecology and Biological Resources [in Vietnamese].

Corbet. G. B., Hill. J. E., 1992. The mammals of the Indomalayan region: a systematic review, Oxford university press Oxford, 488 pp.

Csorba. G., Ujhelyi. P., và Thomas. N., 2003. Horseshoe bats of the world:(Chiroptera: Rhinolophidae). Alana Books, 158.

Tran Hong Hai, 2010. Study on the mammal fauna of Muong Do Nature Reserve, Phu Yen district, Son La Province with recommendations for conservation. $\mathrm{PhD}$ thesis, Institute of Ecology and Biological Resources, Ha Noi, Vietnam.
Tran Hong Hai, Tran Hong Viet, Le Xuan Canh, Nguyen Xuan Dang, 2010. Results of mammal study at Muong Phong, Phu Yen district, Son La Province. Tap chi Sinh hoc, 32(2): 31-42.

Dang Huy Huynh, Cao Van Sung, Dao Van Tien, Pham Trong Anh, Hoang Minh Khien, 1994. Checklist of mammals in Vietnam. Publishing House Science and Technics, Ha Noi: 168 pp.

Kruskop S. V., 2013. Bats of Vietnam Checklist and an identification manual. Joint Russian-Vietnamese Science and Technological Tropical Centre. Moscow. Russia: 316 pp.

Pham Van Nha, 2008. Study on mammals of Son La Province, PhD thesis, Hanoi National University of Education, Ha Noi, Vietnam.

Tran Hong Viet, Tran Hong Hai, Pham Van Nha, 2006a. Results of mammal study in Thuan Chau district, Son La Province. HNUE Journal of Science, 4: 150-158.

Tran Hong Viet, Tran Hong Hai, Pham Van Nha, 2006b. Results of mammal study in Quynh Nhai district, Son La Province. HNUE Journal of Science, 4: 141-149.

Tran Hong Viet, Tran Hong Hai, Pham Van Nha, 2006c. An assessment of the mammal fauna of Moc Chau district, Son La Province with recommendations for conservation. Tap chi Sinh hoc, 28(4): $18-27$.

Tran Hong Viet, Tran Hong Hai, Pham Van Nha, 2006d. First records of mammals from Yen Chau district, Son La Province. HNUE Journal of Science, 1: 146-155

Tran Hong Viet, Tran Hong Hai, Pham Van Nha, 2007a. A check list of mammal from Son La Province. HNUE Journal of Science, 1: 116-125.

Tran Hong Viet, Tran Hong Hai, Pham Van Nha, 2007b. Results of mammals study in Song $\mathrm{Ma}$ and Sop Cop districts, Son La Province. HNUE Journal of Science, 1: 107-115. 